\title{
Use of a small low-noise wind tunnel for determining the aeroacoustic noise produced by components on a vehicle
}

\author{
M J Kingan ${ }^{1 *}$, J R Pearse ${ }^{1}$, and P D Hubbard ${ }^{2}$ \\ ${ }^{1}$ Department of Mechanical Engineering, University of Canterbury, Christchurch, New Zealand \\ ${ }^{2}$ Hubco Automotive Limited, Christchurch, New Zealand
}

The manuscript was received on 27 December 2005 and was accepted after revision for publication on 20 July 2006.

DOI: 10.1243/09544070JAUTO249

\begin{abstract}
This paper describes an experimental method used to determine the aeroacoustic noise produced by a 'roof rack' placed on the roof of a vehicle. Testing was done on a vehicle roof positioned at the outlet jet of a small low-noise wind tunnel. A 'simulated vehicle cabin' was constructed beneath the vehicle roof that had similar absorption characteristics to an actual vehicle cabin. Sound pressure level measurements were made within the simulated vehicle cabin. The sound pressure level measurements were consistent with measurements made within the cabin of an actual vehicle in a large anechoic wind tunnel. The method could also be used to determine the in-cabin aeroacoustic noise produced by other vehicle accessories.
\end{abstract}

Keywords: aeroacoustic, roof rack, automotive noise, wind tunnel

\section{INTRODUCTION}

To meet consumer demand, automotive manufacturers have placed considerable emphasis on reducing in-cabin vehicle noise. Consequently, incabin noise has reduced to a level where aeroacoustic sources, produced by airflow over accessories such as windscreen wipers and roof racks, have become a significant contributor to the noise level inside the cabin, often dominating the in-cabin noise at cruise speeds above $60 \mathrm{mile} / \mathrm{h}$ [1] . To ensure that the aeroacoustic noise produced by airflow over accessories is as low as possible, car manufacturers conduct extensive tests to measure the aeroacoustic noise level produced by these accessories. These tests often involve placing a complete vehicle, with the accessory attached, inside a large anechoic wind tunnel and measuring the sound in the vehicle cabin. Different accessory shapes and positions can be tested to determine the quietest configuration.

* Corresponding author: Department of Mechanical Engineering, University of Canterbury, Private Bag 4800, Christchurch, New Zealand.email: m.kingan@mech.canterbury.ac.nz
Unfortunately, complete vehicle testing in a large anechoic wind tunnel is very expensive. This limits the amount of time that manufacturers are willing to spend conducting complete vehicle tests, which in turn limits the amount of development that can be done with this type of testing.

Owing to the high costs associated with complete vehicle tests, aeroacoustic testing of vehicle accessories is often done in small wind tunnels on isolated components. This is known as isolated component testing. For example, Siegert et al. [2] reported measurements of the aeroacoustic noise produced by a vehicle side mirror using isolated component tests, while Chambers [3] measured the aeroacoustic noise produced by a roof rack and a car radio antenna using isolated component tests.

Chambers [3] noted that 'the feasibility of predicting installed component noise rankings from isolated component test results has not been completely established for small differences in generated noise levels'. Thus, while isolated component testing appears to be useful in the study of the aeroacoustic noise generation mechanisms of an isolated component, it has not been established whether it can 
be used to determine with absolute certainty the in-cabin aeroacoustic noise generated by a component installed on a vehicle.

For example, the level of the aeroacoustic noise produced by an isolated roof rack will not be the same as the aeroacoustic noise produced by an identical roof rack mounted on a vehicle roof, because the airflow over the roof racks is different in each case. Also, by testing on an actual vehicle and measuring the sound level within the cabin, the transmission loss of sound from aeroacoustic sources outside the vehicle is inherently included in the sound level measurements. In the case of roof racks, on-vehicle tests are also useful in determining the optimum location (vertical height and position on the roof) which provides the lowest level of in-cabin noise.

Therefore, in order accurately to determine the in-cabin sound level due to the aeroacoustic noise produced by a roof rack, it is necessary to measure the aeroacoustic noise produced by the roof rack installed on the roof of a vehicle rather than measure the noise produced in an isolated component test. To minimize the cost of testing, it would be ideal to conduct experiments in small, low-cost wind tunnels which are cheaper to construct and operate than the large wind tunnels required for complete vehicle tests.

To measure the aeroacoustic noise produced by a roof rack installed on a vehicle, the roof section of a vehicle (the portion of the vehicle above the height of the bottom of the front windscreen) was placed in the airflow at the exit jet of the low-noise wind tunnel in the Department of Mechanical Engineering at the University of Canterbury, and the sound level was measured by a microphone located in a specially built 'simulated vehicle cabin' beneath the roof section. As this testing was done on a 'partial vehicle' it will be referred to as 'partial vehicle testing', and the roof section of the vehicle used in these tests will be referred to as the 'vehicle roof'.

A specially designed flow guide positioned above the vehicle roof ensured that the airflow over the vehicle roof closely matched that over a complete vehicle. A 'simulated vehicle cabin' was built underneath the vehicle roof that had a similar acoustic environment to the cabin of the actual vehicle. Sound pressure level measurements were made inside the simulated vehicle cabin. The wind tunnel airflow speed was set to simulate a vehicle travelling at $25.5 \mathrm{~m} / \mathrm{s}(57.0 \mathrm{mile} / \mathrm{h})$, and the sound pressure level within the simulated vehicle cabin was measured with and without roof racks mounted on the vehicle roof. The sound pressure level measurements were compared with sound pressure level measurements taken from a complete vehicle test on a similar vehicle.

It is worth mentioning that turbulent pressure fluctuations from the wake of a roof rack may impinge on the vehicle roof and produce noise (for an excellent discussion of the noise generated by separated flow impinging on a vehicle surface, the reader is referred to the work by George [1]). For the partial vehicle tests presented here, the roof rack was situated sufficiently high above the car roof for the wake not to impinge on the car roof. This was confirmed using 'tufts' [4] and an 'oil-film method' [5]. For the tuft investigation a row of $20 \mathrm{~mm}$ long cotton 'tufts' were attached along the centre-line of the roof. During the partial vehicle tests the cotton tufts remained relatively stationary, indicating that the turbulent wake from the roof racks did not impinge upon the vehicle roof. For the 'oil film method' investigation a thin film of paraffin oil containing a suspension of $\mathrm{TiO}_{2}$ particles was 'painted' onto the roof surface downstream of the roof racks. During the partial vehicle tests, no disruption of the oil film was observed, indicating that a turbulent wake was not impinging upon the roof.

Airflow over the roof rack will produce aeroacoustic noise which propagates through the air to the vehicle and is transmitted through the vehicle's structure to the vehicle cabin. However, vibration caused by the airflow over the roof rack will be transmitted down the vehicle structure, which will radiate as sound into the cabin. This source of noise will be inherently included in any on-vehicle tests (full vehicle or partial vehicle tests) but is of course not measured in isolated component tests. Owing to the high rigidity of the roof rack, this method of noise production was assumed to be negligible for the cases presented here.

\section{METHOD}

\subsection{Description of the vehicle roof}

The upper section of a vehicle was obtained. This was cut from a complete vehicle at the bottom of the window pillars retaining the interior roof lining. Consequently, the sound transmission loss through the vehicle roof was identical to that of a complete vehicle. The windows were replaced with $6 \mathrm{~mm}$ thick plywood. The vehicle roof was installed at the exit of the low noise wind tunnel with the bottom of the front windscreen positioned in line with the floor (bottom surface) of the wind tunnel exit nozzle. 


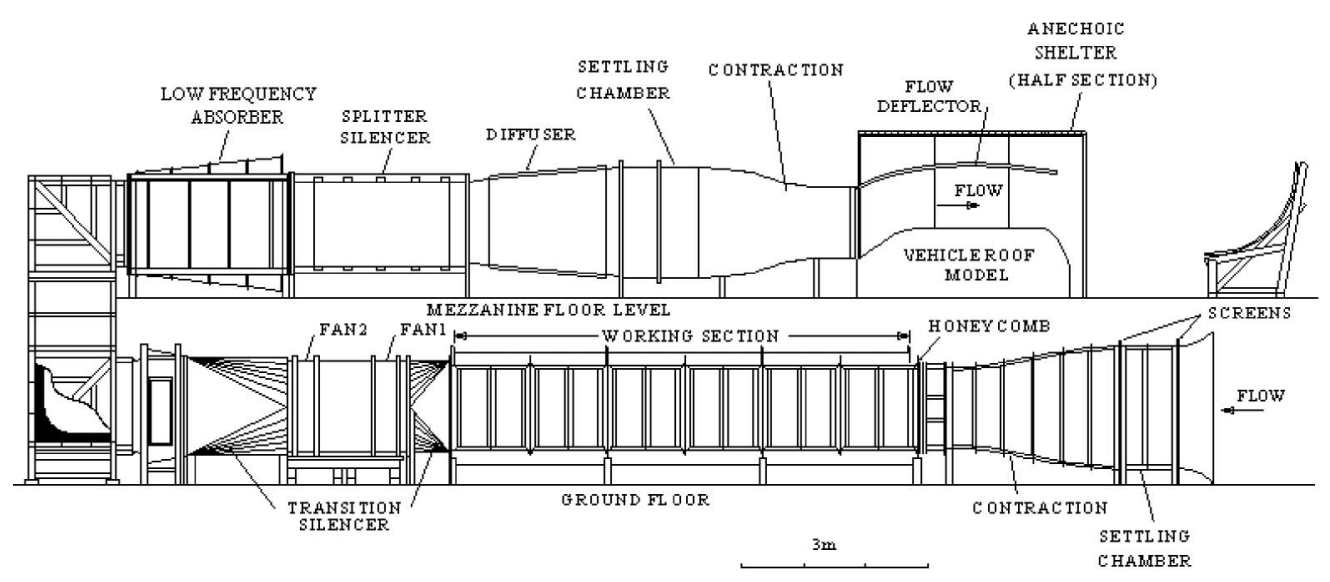

Fig. 1 Wind tunnel layout

\subsection{Wind tunnel arrangement}

The wind tunnel is an open circuit type wind tunnel capable of airflow speeds at the outlet jet of $25.5 \mathrm{~m} / \mathrm{s}$ in the configuration used here. The wind tunnel is described in detail in reference [6] and a schematic showing the wind tunnel arrangement for the partial vehicle tests is shown in Fig. 1.

For the work described in this paper, a new two-dimensional contraction was designed and built to accommodate the vehicle roof. With the configuration used here, airflow passed from a $1.53 \mathrm{~m} \times$ $1.53 \mathrm{~m}$ cross-section settling chamber to the twodimensional contraction from which the airflow exited the wind tunnel. The two-dimensional contraction restricted the flow in only the vertical direction to give an exit nozzle size of $0.77 \mathrm{~m} \times$ $1.53 \mathrm{~m}$. The inner surfaces of the contraction were lined with $50 \mathrm{~mm}$ thick sound absorbent foam to help reduce the wind tunnel background noise level. A schematic showing the settling chamber and twodimensional contraction of the modified low-noise tunnel is shown in Fig. 2.

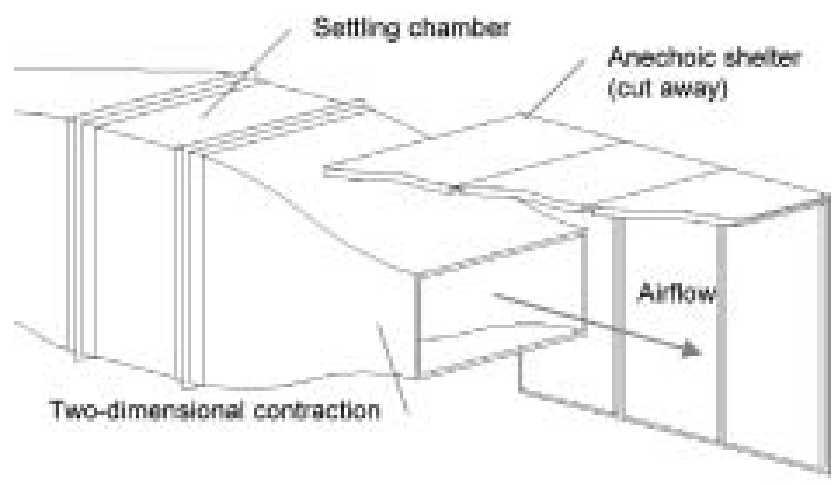

Fig. 2 Settling chamber and two-dimensional contraction of the low-noise wind tunnel

\subsection{Flow guide design}

The airflow over a complete vehicle was simulated using a commercial computational fluid dynamics (CFD) software package. From this simulation the streamlines above the roof of the vehicle were determined. A streamline above the vehicle roof along the vehicle centre-line defined the shape and position of a two-dimensional rigid flow guide positioned above the vehicle roof which ensured that the airflow closely matched that over a complete vehicle.

The flow guide was constructed from $6 \mathrm{~mm}$ plywood and extended almost to the walls of the anechoic shelter. To prevent a standing wave forming between the car roof and the flow guide, the flow guide was lined with $10 \mathrm{~mm}$ thick sound-absorbing material.

As there is no flow across a streamline, positioning a rigid flow deflector along one of the streamlines predicted by the CFD simulation helped 'force' the airflow over the vehicle roof to match that over the complete vehicle. Strictly, for the (potential) airflow over the vehicle roof to match the airflow over a complete vehicle, the inlet and outlet conditions must also be identical. This was not verified. However, the good correspondence of the measured velocity profile to the velocity profile predicted by the CFD simulation indicated that the airflow over the vehicle roof would likely be very similar to that over a complete vehicle.

The CFD results confirmed that the flow over the car roof was approximately two-dimensional (i.e. did not vary significantly across the width of the vehicle roof). Therefore, the two-dimensional shape of the flow deflector should force the correct flow over the width of the vehicle roof.

As the dimensions of the roof rack are small compared with the dimensions of the vehicle roof and 
with the height to the flow deflector above the vehicle roof, it was assumed that the presence of the roof rack would not significantly affect the free-streamline shape (and thus the shape of the flow deflector) above the vehicle roof model.

\subsection{Comparison of the airflow over the vehicle roof with CFD results}

The velocity profile above the position of the first roof rack $(900 \mathrm{~mm}$ downstream of the top of the front windscreen), along the centre of the car, was measured using a pitot tube and compared with that predicted by the CFD simulation. If the airflow over the vehicle roof and the CFD model is similar, then

$$
\frac{u_{\mathrm{e}}(y)}{U_{\mathrm{e}}}=\frac{u_{\mathrm{f}}(y)}{U_{\mathrm{f}}}
$$

where $u_{\mathrm{f}}(y)$ is the velocity profile predicted by the CFD simulation, $u_{\mathrm{e}}(y)$ is the experimental velocity profile, $U_{\mathrm{f}}$ is the simulated vehicle speed in the CFD simulation $\left(U_{\mathrm{f}}=30 \mathrm{~m} / \mathrm{s}\right), U_{\mathrm{e}}$ is the simulated vehicle speed in the experiment, and $y$ is the vertical coordinate above the vehicle roof.

Figure 3 compares the experimental velocity profile $u_{\mathrm{e}}(y) / U_{\mathrm{e}}$, with the velocity profile predicted by the CFD simulation $u_{\mathrm{f}}(y) / U_{\mathrm{f}}$. The good agreement of the experimental and CFD velocity profiles indicates that the airflow over the vehicle roof in the wind tunnel matches the airflow over the actual vehicle roof very well. The calculated value of $U_{\mathrm{e}}$ for this case was $25.5 \mathrm{~m} / \mathrm{s}$ with the wind tunnel running at its maximum airflow speed.

\subsection{Matching the acoustic absorption of the vehicle cabin and the simulated vehicle cabin}

A simulated vehicle cabin was designed and built beneath the vehicle roof that was approximately the same volume and had similar reverberation times to the cabin of an actual vehicle. This ensured that sound pressure level measurements made inside the simulated vehicle cabin were comparable with those made within the cabin of an actual vehicle.

To ensure that the sound absorption in the simulated vehicle cabin was similar to the sound absorption in the actual vehicle cabin, sound absorption was added to the floor and walls of the simulated vehicle cabin until the reverberation time within the cabin matched that of an actual vehicle cabin. Care was taken to ensure that the sound-absorbing material was evenly spread throughout the cabin. The mean $T_{20}$ reverberation times of the simulated vehicle cabin and the actual vehicle cabin are plotted in Fig. 4.

The reverberation times within the simulated vehicle cabin were very similar to the reverberation times within the actual vehicle cabin for frequencies higher than $100 \mathrm{~Hz}$. Thus, sound pressure level measurements made inside the simulated vehicle cabin were expected to be comparable with those made within the cabin of an actual vehicle for frequencies higher than $100 \mathrm{~Hz}$.

\subsection{Experimental arrangement}

The vehicle roof was positioned at the exit of the wind tunnel (see Fig. 1) with the front edge of the windscreen positioned flush against the wind tunnel floor. The flow guide was installed above the vehicle roof. Sound pressure level measurements within the simulated vehicle cabin were made at a position that corresponded approximately to the position of the driver's head in an actual vehicle. The roof racks were installed on the vehicle roof with the leading edge of the front roof rack positioned $900 \mathrm{~mm}$ from the top of the front windscreen and the leading edge of the

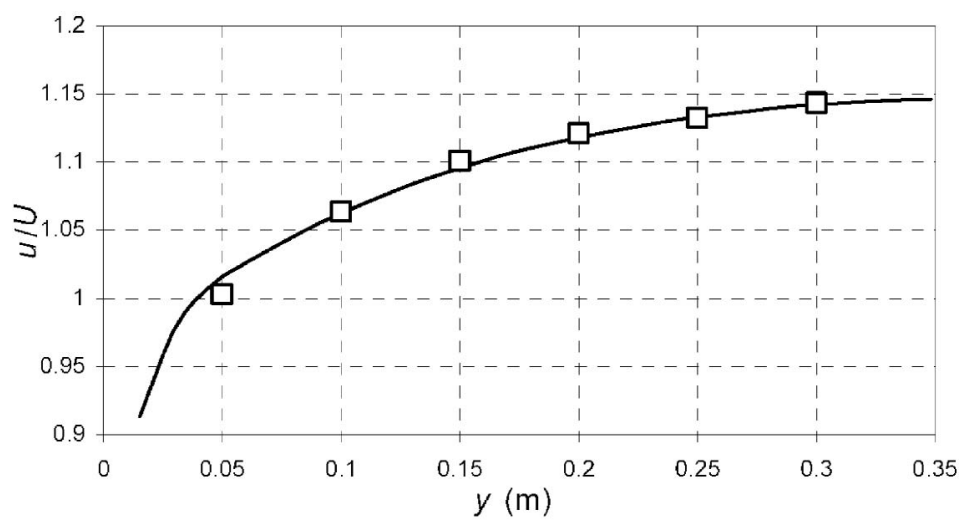

Fig. 3 Velocity profile above the vehicle roof at the position of the front roof rack $900 \mathrm{~mm}$ from the top of the front windscreen), along the vehicle centreline: $\square$, experimental velocity profile $u_{\mathrm{e}}(y) / U_{\mathrm{e}} ;$, velocity profile predicted by the CFD simulation $u_{\mathrm{f}}(y) / U_{\mathrm{f}}$ 


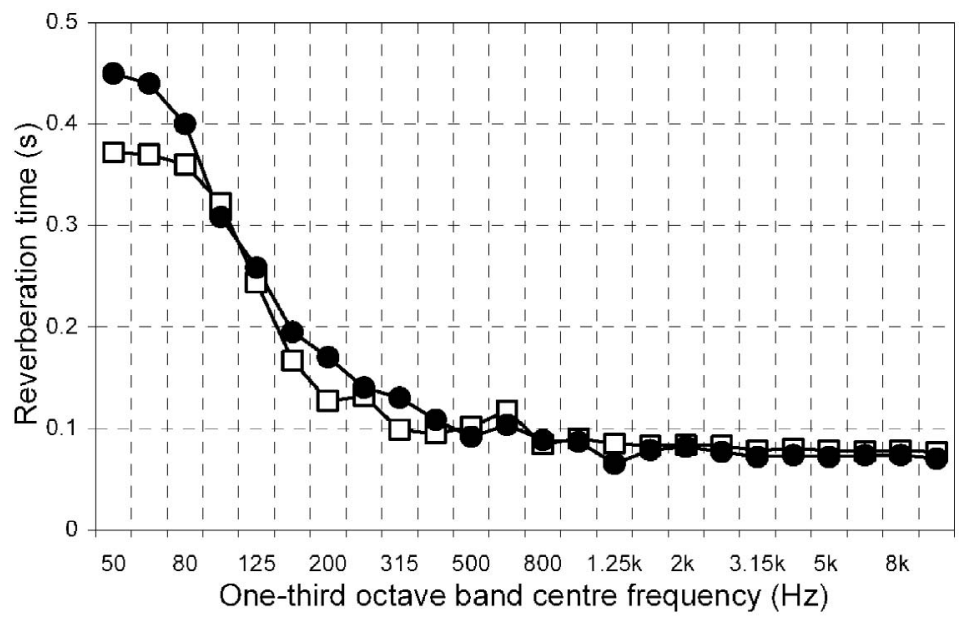

Fig. 4 Mean $T_{20}$ reverberation time: $-\bullet$, measured in an actual vehicle cabin; $-\square-$, measured in the simulated vehicle cabin

rear roof rack positioned $870 \mathrm{~mm}$ behind the leading edge of the front roof rack.

\section{RESULTS AND DISCUSSION}

In this section the results of the testing on the vehicle roof, which is referred to as the partial vehicle test, are discussed. These results are compared with the results of a complete vehicle test on a similar vehicle. The complete vehicle tests were conducted independently by an automotive manufacturer in a large anechoic wind tunnel.

Figure 5 shows the sound pressure level, $L$, measured in the cabin in the complete vehicle test, at approximately the position of a driver's ear, with and without roof racks. The wind tunnel airflow speed for this test was $27.8 \mathrm{~m} / \mathrm{s}$.

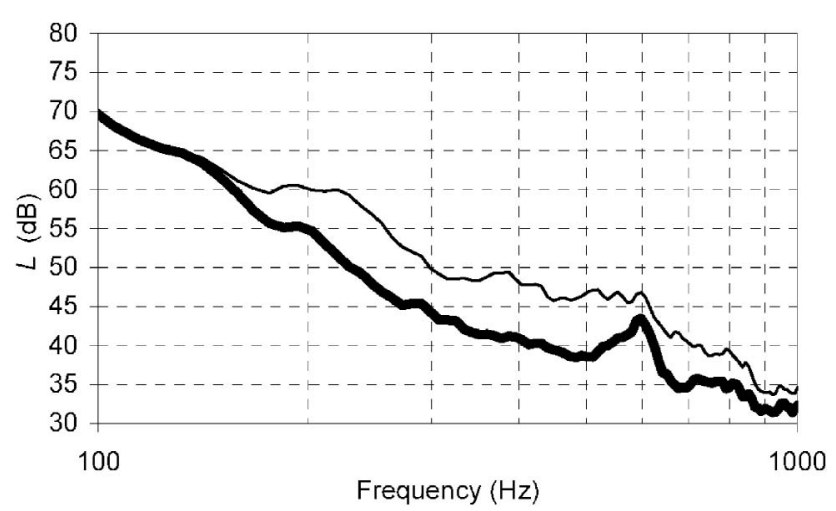

Fig. 5 Complete vehicle test: sound pressure level measured in the cabin of a complete vehicle. The airflow speed was $27.8 \mathrm{~m} / \mathrm{s}$ : - , $L$ without roof racks installed on the vehicle roof;,$- L$ with roof racks installed on the vehicle roof
Figure 6 shows the sound pressure level measured in the partial vehicle test at approximately the driver's ear with and without roof racks. The wind tunnel airflow speed corresponded to a car travelling at $25.5 \mathrm{~m} / \mathrm{s}$.

For both tests a corrected sound pressure level, $L_{\mathrm{c}}$, is defined

$$
\begin{array}{ll}
L_{\mathrm{c}}=10 \log _{10}\left(10^{L_{\mathrm{r}} / 10}-10^{L_{\mathrm{v}} / 10}\right), & L_{\mathrm{r}}-L_{\mathrm{v}} \geqslant 2 \\
L_{\mathrm{c}}=\text { undefined }, & L_{\mathrm{c}}-L_{\mathrm{v}}<2
\end{array}
$$

where $L_{\mathrm{r}}$ is the sound pressure level measured with roof racks and $L_{\mathrm{v}}$ is the sound pressure level measured without roof racks. Essentially, $L_{\mathrm{c}}$ is a measure of the noise generated by the roof racks (installed on the vehicle roof), which should be independent of the background noise level.

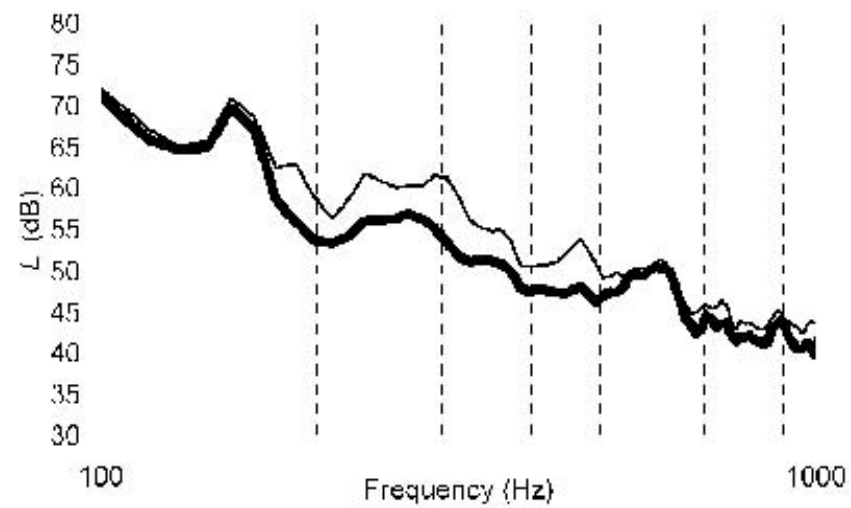

Fig. 6 Partial vehicle test: $L$ measured in the simulated vehicle cabin. The airflow speed corresponds to a car travelling at $25.5 \mathrm{~m} / \mathrm{s}:-, L$ without roof racks attached to the vehicle roof;,$- L$ with roof racks attached to the vehicle roof 
George [7] suggests the following scaling law for vehicles that are the same size but for which the airflow speeds are different

$$
L_{\mathrm{cu}}=L_{\mathrm{c}}-10 n \log _{10}\left(\frac{U_{\mathrm{e}}}{U_{\mathrm{ref}}}\right)
$$

where $U_{\text {ref }}$ is a reference velocity (taken to be $1 \mathrm{~m} / \mathrm{s}$ ) and $n$ is a velocity dependence exponent. Because of the low Mach number of the tests $(M \ll 1)$, and assuming that the roof rack is rigid (i.e. does not vibrate), the aeroacoustic noise produced will be 'dipole type' (see reference [1]) for which the radiated mean square sound pressure should scale in proportion to $U_{\mathrm{e}}^{6}$ (similar to that radiated by a circular cylinder in a cross-flow - see, for example, reference [8]). Thus, for the analysis presented here it is assumed that $n=6$.

According to George [7], the frequency should scale according to a Strouhal number type relationship

$$
S t=\frac{f D}{U_{\mathrm{e}}}
$$

where $D$ is a reference length (taken to be $0.2 \mathrm{~m}$ ). The normalized sound pressure levels, $L_{\mathrm{cu}}$, of the partial vehicle test and the full vehicle test are plotted against Strouhal number $S t$ in Fig. 7. Below $500 \mathrm{~Hz}$ there was reasonable agreement between the calculated $L_{\mathrm{cu}}$ values, which indicated that the partial vehicle method provided an accurate indication of the in-cabin sound pressure level due to the aeroacoustic noise produced by airflow over the roof racks.

In the partial vehicle test above $500 \mathrm{~Hz}$, the sound pressure level produced for the case with the roof rack was generally less than $2 \mathrm{~dB}$ above the sound pressure level produced for the case without

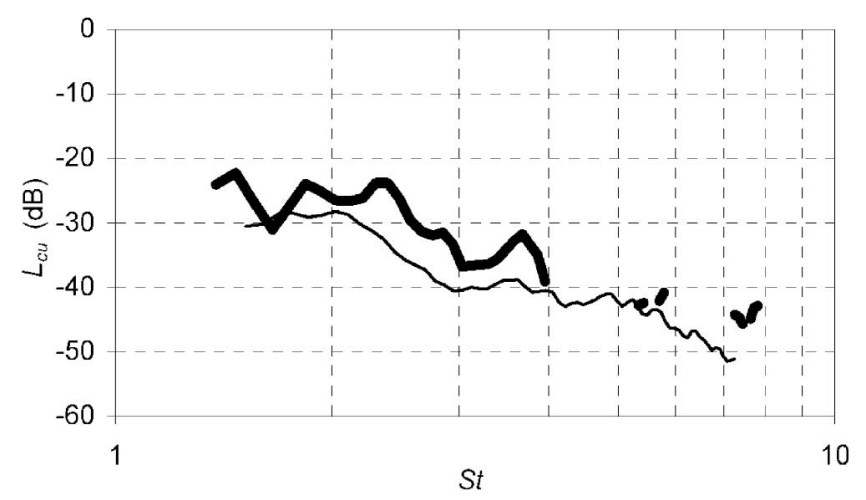

Fig. 7 Corrected sound pressure level $L_{\text {cu }}$ : -, complete vehicle test; - , partial vehicle test the roof rack (see Fig. 7). This meant that $L_{\mathrm{c}}$ (and consequently also $L_{\mathrm{cu}}$ ) was mostly undefined for frequencies greater than $500 \mathrm{~Hz}$. This was most probably due to the relatively higher wind tunnel background noise level in the small low-noise wind tunnel, which increased the sound pressure level inside the simulated vehicle cabin to a level close to that produced by the car roof racks. This resulted in $L_{\mathrm{c}}$ (and $L_{\mathrm{cu}}$ ) being undefined for most frequencies above $500 \mathrm{~Hz}$. Reduction in the wind tunnel background noise level at these frequencies would thus allow measurements to be made over a wider frequency range.

\section{CONCLUSIONS}

This paper has described a method for measuring the aeroacoustic noise produced by roof racks installed on the roof of a vehicle. Tests were undertaken on a vehicle roof installed at the exit of a low-noise wind tunnel. Sound pressure level measurements were made in a simulated vehicle cabin beneath the vehicle roof.

Measurements confirmed that the airflow over the vehicle roof was similar to that over a complete vehicle. Treatments were applied to the simulated vehicle cabin to make the acoustic environment within the cabin similar to the interior of a complete vehicle. This ensured that sound pressure level measurements inside the simulated vehicle cabin were made in a similar environment to those made in a complete vehicle test on a similar vehicle in a large anechoic wind tunnel.

The sound pressure level at a position inside the simulated vehicle cabin was measured with and without roof racks installed. The wind tunnel airflow speed simulated the airflow over the roof of a vehicle travelling at $25.5 \mathrm{~m} / \mathrm{s}$. The measured sound pressure level was corrected for airflow speed and bare vehicle noise and compared with corrected sound pressure level measurements made in a complete vehicle test on a similar vehicle in a large wind tunnel.

Below $500 \mathrm{~Hz}$ the corrected sound pressure level measured in the partial vehicle test was reasonably similar to the corrected sound pressure level measured in the complete vehicle test, indicating that partial vehicle tests could be used to measure the aeroacoustic noise produced by an accessory installed on a vehicle. However, comparison of a partial vehicle test with an equivalent complete vehicle test (i.e. same vehicle type and airflow speed) would be required to confirm this. 
The high background noise levels above $500 \mathrm{~Hz}$ for the partial vehicle tests meant that the results of the partial and complete vehicle tests could not be compared for frequencies higher than $500 \mathrm{~Hz}$. Reduction in the wind tunnel background noise would be necessary to compare the sound pressure level produced by roof racks at these frequencies.

\section{ACKNOWLEDGEMENTS}

The authors would like to acknowledge the support of Hubco Automotive and the New Zealand Foundation for Research, Science, and Technology.

\section{REFERENCES}

1 George, A. R. Automobile aerodynamic noise. SAE special publication 900315, 1990.

2 Siegert, R., Schwarz, V., and Reichenberger, J. Simulation of aeroacoustic sound generated by generic bodies placed on a plate - prediction of radiated sound pressure. AIAA paper 99-1895, 1999.

3 Chambers, F. Isolated component testing for the identification of automotive wind noise sources.
Winter Annual Meeting of the American Society of Mechanical Engineers, Dallas, Texas, 25-30 November 1990.

4 Crowder, J. P. Tufts. In Handbook of flow visualization (Ed. W.-J. Yang), 1989, p. 125.

5 Reznicek, R. Surface tracing methods. In Handbook of flow visualization (Ed. W.-J. Yang), 1989, pp. 91-103.

6 Kingan, M. J. and Pearse, J. R. Development of a low noise wind tunnel. Internoise 2005, Rio de Janeiro, Brazil, 7-10 August 2005, paper 2090.

7 George, A. R. Automobile wind noise and its measurement. SAE special publication 1184, 1996.

8 Blake, W. K. Mechanics of glow-induced sound and vibration. Vol. 1. General concepts and elementary sources, Applied Mathematics and Mechanics, vol. 17-1, 1986, Ch. 4 (Academic Press, Orlanda, Florida).

\section{APPENDIX}

\section{Definitions}

Roof rack: carrier system mounted on the roof of a vehicle for holding items such as bicycles, kayaks, skis, and roof boxes.

$T_{20}$ reverberation time: time for the sound level within a space to decay $60 \mathrm{~dB}$, estimated from the length of time for the sound level to decay $20 \mathrm{~dB}$ 